\section{OP0299 IN RHEUMATOID ARTHRITIS PATIENTS HIGHER NUMBER OF COMORBIDITIES PREDICTS 6-MONTH INSUFFICIENT RESPONSE TO FIRST BIOLOGIC THERAPY AND EVENTUAL CATEGORIZATION OF THE DISEASE AS DIFFICULT-TO-TREAT}

I. Flouri ${ }^{1}$, A. Repa ${ }^{1}$, N. Avgustidis ${ }^{1}$, N. Kougkas ${ }^{1}$, A. Eskitzis ${ }^{1}$, A. Molla Ismail Sali ${ }^{1}$, S. Pitsigavdaki ${ }^{1}$, K. Pateromichelaki ${ }^{1}$, E. Kalogiannaki ${ }^{1}$, M. Terizaki ${ }^{1}$, G. Bertsias ${ }^{1}$, P. Sidiropoulos ${ }^{1} .{ }^{1}$ University Hospital of Heraklion, Crete, Rheumatology Department, Heraklion, Crete, Greece

Background: Difficult-to-treat rheumatoid arthritis (D2T RA) was recently defined by a EULAR study group (1) and, as a disease category it is largely complicated and under-researched. Patient comorbidities may play a significant role in the response to therapy with biologic disease-modifying antirheumatic drugs (bDMARDs) and in the disease classification as D2T RA.

Objectives: To evaluate the impact of comorbidities [studied as total Comorbidities Count (CC) and rheumatic disease comorbidity index (RDCl)] on 6-month response to therapy with the first bDMARD in real-world clinical practice and on eventual disease designation as D2T RA.

Methods: Prospective study of all RA patients who start any bDMARD in a tertiary centre University Hospital after their consent. All patient comorbidities [among a list of approximately 100 pre-specified major comorbidities] are registered by treating physicians. Response to therapy was defined as achievement of low disease activity or remission (LDA/Rem) according to simplified disease activity index (SDAl) and health assessment questionnaire (HAQ) improvement of $\geq 0.25$.

D2T RA patient group was defined according to the EULAR definition of D2T RA and was compared to: $a /$ all other patients and $b / t$ to a sub-group of patients designated as "well-controlled RA" (follow-up $\geq 2$ years and $\geq 2$ visits in the last year in LDA/Rem).

Logistic regression models were used to adjust for the potential confounding of age, sex, disease duration, seropositivity, number of previous synthetic DMARDs, type of $1^{\text {st }}$ bDMARD initiated (TNF inhibitor vs. non-TNF inhibitor), co-administered methotrexate and corticosteroids (yes/no), baseline SDAI and $\mathrm{HAQ}$ and year of therapy start.

Results: Analysis included 501 RA patients who received a total of 1098 bDMARD treatments. At $1^{\text {st }}$ bDMARD treatment start, patients (women: $81 \%$ ) had a median (IQR) age: 60 (51-68) years, disease duration: 5.4 (3-11) years, SDAl: 36 (28-46), HAQ: 1.0 (0.5-1.5), CC: 3 (2-6) kal RDCl: 2 (0-3). In adjusted analyses, total comorbidity count $(C C) \leq 1$ (vs $\geq 2$ ) was predicting LDA/Rem at 6 months of therapy [OR $(95 \% \mathrm{Cl})=4.1(1.5-11), \mathrm{p}=0.005]$, while $\mathrm{RDCl}=0$ (vs. $\geq 1$ ) was predicting HAQ improvement $\geq 0.25[\mathrm{OR}(95 \% \mathrm{Cl})=2.6$ (1.2-6.7), $\mathrm{p}=0.046]$

During 2614 patient-years of follow-up, the disease in 98 patients could be classified as "D2T RA", while 127 patients had "well-controlled RA" Baseline independent predictors for D2T RA compared to all other patients were $\mathrm{RDCl} \geq 1$ (vs. $0)[\mathrm{OR}=3.3(1.7-9.4), \mathrm{p}=0.024]$, female sex $[\mathrm{OR}=3.1(1.01-9.5)]$ and age [OR $=0.97(0.94-0.99)]$. Multivariable analyses for predictors of "D2T" compared to "well-controlled" RA yielded similar results.

Conclusion: In RA patients starting the first bDMARD treatment, a higher number of comorbidities at baseline is an independent predictor of lower 6-month response to therapy and final disease classification as "difficult-to-treat" RA. REFERENCES:

[1] Nagy G, Roodenrijs NM, Welsing PM, Kedves M, Hamar A, van der Goes $M C$, et al. EULAR definition of difficult-to-treat rheumatoid arthritis. Ann Rheum Dis. 2021 Jan;80(1):31-5.

Acknowledgements: Pancretan Health Association and Special Account for Research Grants (ELKE) - University of Crete.

Disclosure of Interests: None declared.

DOI: 10.1136/annrheumdis-2021-eular.4110

\section{Quantitative imaging}

\section{OP0300 USE OF THERMOGRAPHY OF HANDS AND MACHINE LEARNING TO QUANTIFY JOINT INFLAMMATION AND ESTIMATE DAS28, CDAI, SDAI IN PATIENTS WITH RHEUMATOID ARTHRITIS}

I. Morales-Ivorra ${ }^{1,2}$, C. Gómez Vaquero ${ }^{3}$, C. Moragues Pastor ${ }^{3}$, J. M. Nolla ${ }^{3}$, J. Narváez ${ }^{3}$, J. A. Narvaez ${ }^{4}$, D. Grados Canovas ${ }^{1}$, M. A. Marin-López ${ }^{2} .{ }^{1}$ Hospital
Igualada, Rheumatology, Igualada, Spain; ${ }^{2}$ Singularity Biomed, $R+D$, Sant Cugat del Vallès, Spain; ${ }^{3}$ Hospital Universitari de Bellvitge, Rheumatology, L' Hospitalet de Llobregat, Spain; ${ }^{4}$ Hospital Universitari de Bellvitge, Radiodiagnosis, L' Hospitalet de Llobregat, Spain

Background: Disease activity scores such as DAS28, CDAI and SDAI are used in the follow-up of patients with rheumatoid arthritis (RA). These scores include variables obtained on physical examination such as the tender joint count (TJC) and the swollen joint count (SJC). In telematic consultations, it is not possible to determine these variables by physical joint assessment. Therefore, it is necessary to develop new tools that allow detecting joint inflammation in places close to the patient. Thermography is a safe and fast technique that measures heat through infrared imaging. Inflammation of the joints causes an increase in temperature and can therefore be detect by thermography. Machine learning methods are highly accurate in analyzing medical images automatically.

Objectives: To develop an algorithm that, based on thermographic images of hands and machine learning, learn to quantify joint inflammation in patients with RA and estimate the DAS28, CDAI, SDAI by including the patient global health (PGH).

Methods: Multicenter observational study conducted in the rheumatology and radiology service of two hospitals. Patients with RA, psoriatic arthritis (PA), undifferentiated arthritis (UA) and arthritis of hands secondary to other diseases (SA) that attended the follow-up visits were recruited. Companions of patients and healthcare professionals were also recruited as healthy subjects (HS). In all cases, a thermographic image of the hands was taken using a Flir One Pro or a Thermal Expert TE-Q1 camera connected to a smartphone. Ultrasound (US) of both hands was performed in patients with RA, PA, UA and SA. The degree of synovial hypertrophy (SH) and power doppler (PD) was assessed for each joint (score from 0 to 3 ). Machine learning was used to quantify joint inflammation ( $\mathrm{SH}+\mathrm{PD}$ ) from the thermal images using US as ground truth. RA patients whose thermal image was taken with the Thermal Expert TE-Q1 camera were used to evaluate the performance (test dataset). The other participants were used as training dataset. The TJC, SJC, PGH, C-reactive protein (CRP) and erythrocyte sedimentation rate (ESR) were also assessed in the test dataset. $A$ linear regression was used to estimate the DAS28, CDAI and SDAl with the resultant joint inflammation quantification from the thermal images and the PGH. Performance was evaluated by means of Pearson's correlation coefficient. The study was approved by the Clinical Ethics and Research Committee of both centers.

Results: The total number of recruited subjects was 521 (422 for the training and 99 for the testing dataset). In the training dataset, the thermography of 296 patients was taken with the Flir One Pro (163 RA, 17 PA, 22 UA, 12 SA and 82 HS) and 126 with the Thermal Expert TE-Q1 camera (6 RA without clinical data, $20 \mathrm{PA}, 7 \mathrm{UA}, 23 \mathrm{SA}$ and $70 \mathrm{HS}$ ).

We found higher correlations between joint inflammation variables (US and SJC) and thermography $(0.48, p<0.01$ for US and $0.48, p<0.01$ for SJC) than between joint inflammation variables (US and SJC) and the PGH $(0.29, p<0.01$ for US and $0.35, p<0.01$ for $\mathrm{SJC}$ ). Thermography did not show statistically significant correlation with the $\mathrm{PGH}(0.14, \mathrm{p}=0.164)$. The linear regression of thermography and the PGH showed strong correlation with the DAS28 $(0.73, \mathrm{p}<0.01)$, CDAI $(0.84$ $\mathrm{p}<0.01)$ and $\operatorname{SDAI}(0.82, \mathrm{p}<0.01)$.

Conclusion: Thermography of hands and machine learning can effectively quantify joint inflammation and can be used in combination with the $\mathrm{PGH}$ to estimate disease activity scores. These results open an opportunity to develop tools that facilitate telematic consultations in patients with RA.

\section{REFERENCES:}

[1] Brenner M, Braun C, Oster M, Gulko PS. Thermal signature analysis as a novel method for evaluating inflammatory arthritis activity. Ann Rheum Dis. 2006;65(3):306-11

[2] Lynch CJ, Liston C. New machine-learning technologies for computer-aided diagnosis. Nat Med. 2018;24(9):1304-1305

[3] Tan YK, Hong C, Li H, Allen JC Jr, Thumboo J. Thermography in rheumatoid arthritis: a comparison with ultrasonography and clinical joint assessment. Clin Radiol. 2020;75(12):963

Disclosure of Interests: None declared.

DOI: 10.1136/annrheumdis-2021-eular.1247 